\title{
Influence of Demethylation Pathway on the Differentiation Potential of Human Mesenchymal Stem Cells
}

\section{Faisal Ali ${ }^{1,2 *}$, Yazan Ranneh ${ }^{1}$, Amin Ismail $^{1}$ and Bart Vaes ${ }^{2}$}

${ }^{1}$ Department of Nutrition and Dietetics, Faculty of Medicine and Health Sciences, Nutrigenomics Programme, Universiti Putra Malaysia, 43400 UPM Serdang, Selangor Malaysia

${ }^{2}$ Metabolism and genomics group, Division of Human Nutrition, Wageningen University, Bomenweg 2, 6703 HD Wageningen, The Netherlands

\begin{abstract}
Osteoporosis is associated with a decrease in the commitment of Mesenchymal stem cells (MSC) to the bone forming osteoblast lineage and an increase in the commitment to the fat forming adipocyte lineage in bone marrow of elderly persons. A link between methylation pathway and MSC differentiation remains unclear. Therefore, we hypothesize that alterations in the commitment and differentiation potential of MSC during osteoporosis may be mediated by modification of global methylation pathway. To examine the role of methylation pathway on the differentiation potential of MSC into osteoblasts or adipocytes, human MSC, were used. Adox, which mimics hyperhomocysteinemia effect, was added to cells as a potent methylation inhibitor to block the global methylation pathway of DNA, RNA, Lipid and protein. The effect of demethylation on osteoblast differentiation was determined by measuring the alkaline phosphates activity and the degree of calcification. While, adipocyte differentiation was determined by Oil-red $\mathrm{O}$ staining and triglyceride content. It was clearly observed that demethylation reduces alkaline phosphates activity, calcification and thereby, osteoblast differentiation. In contrast, adipocyte differentiation was stimulated by demethylation. The results of this study suggest that demethylation changes the differentiation potential of MSC for more adipogenic and less osteogenic.
\end{abstract}

Keywords: MSC; Methylation pathway; Osteablast; Adipocyte; Osteoporosis; Adox

\section{Introduction}

Homocysteine (Hcy) is a non-protein forming amino-acid derived from methionine via methylation cycle, which is dependent on the level of vitamin B12. The latter is a cofactor of Hcy metabolism to re-synthesize methionine in the so called remethylation pathway and takes place in all cells. Thus, elevated circulating Hcy in adults is mainly caused by vitamin B12 deficiency [1]. Little is known about the mechanistic role of hyperhomocysteinemia and/or low vitamin B12 in osteoporosis. One of the explanations is that elevated Hcy and low vitamin $\mathrm{B} 12$ based on recent studies have been correlated with bone resorption via stimulation of osteoclast activity [2-4]. But so far the relationship between osteoblst activity and function in the presence of high Hcy and low vitamin B12 levels has been not completely understood. However, recently [5] reported that accumulation of Hcy by decreasing concentrations of vitamin B12 does not influence the activity of human osteoblasts in vitro. Furthermore, most of the previous studies on the pathogenicity of homocysteine have mainly focused on the direct effects of the amino acid [6-8]. However, elevated level of homocystein may be an indirect effect for a disturbance in the methylation cycle [9-11]. In this cycle, Hcy is remethylated to an essential amino acid methionine by the donation of a methyl group from 5-methyl THF, as shown in Figure 1. The remethylation of Hcy occurs by the enzyme methionine synthase (MS), the enzyme MS requires vitamin B12 as cofactor that mediates the transfer of a methyl group under normal physiologic conditions $[12,13]$.

Methionine is then converted to S-adenosylmethionine (SAM) by the enzyme methionine adenosyltransferase (MAT). SAM is the major biological methyl group donor to a large variety of acceptor substrates, including DNA, RNA, phospholipids, and proteins. S-adenosylhomocysteine (SAH) is formed, through donation of a methyl group, which is subsequently hydrolyzed to Hcy by the enzyme $\mathrm{SAH}$ hydrolase $(\mathrm{SAHH})[14,15]$. The ratio SAM/SAH is an important metabolic marker for cellular methylation status and also known methylation potential (MP) [16]. Previous studies in vitro have been reported that inhibition of SAHH enzyme results in decreased SAM levels as a result of decreased methyltransferase activity leading to reduced SAM/SAH ratio in presence of significant increase in SAH levels $[17,18]$. Furthermore, an altered SAM/SAH ratio by inhibition

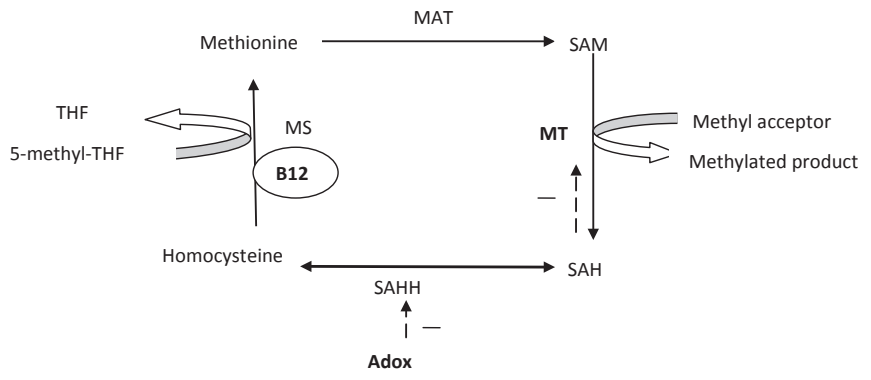

Figure 1: Methylation cycle and inhibitors (dotted line).

Abbreviations: MS: methionine synthase, SAHH: Sadenosylhomocysteine Hydrolase, MAT: methionine adenosyltransferase, THF: tetrahydrofolate, SAM: S-adenosylmethionine, SAH: S-adenosylhomocysteine, methyl acceptor can indicate DNA, RNA, protein or lipid. MT: methyltransferase. Adox: periodate oxidized adenosine as methylation inhibitor for $\mathrm{SAHH}$.

*Corresponding author: Faisal Ali, Department of Nutrition and Dietetics, Faculty of Medicine and Health Sciences, Nutrigenomics Programme, Universiti Putra Malaysia, 43400 UPM Serdang, Selangor, Malaysia, Tel: +60172217485; Fax: + 60389426769; E-mail: sshrmany@yahoo.co.uk

Received September 03, 2013; Accepted September 21, 2013; Published September 23, 2013

Citation: Ali F, Ranneh Y, Ismail A, Vaes B (2013) Influence of Demethylation Pathway on the Differentiation Potential of Human Mesenchymal Stem Cells. J Stem Cell Res Ther 3: 153. doi:10.4172/2157-7633.1000153

Copyright: ( 2013 Ali F, et al. This is an open-access article distributed under the terms of the Creative Commons Attribution License, which permits unrestricted use, distribution, and reproduction in any medium, provided the original author and source are credited. 
of SAH hydrolase results in inhibition of DNA, RNA, protein and lipid methylation [19]. Thus, considering the crucial role of methylation in various cellular processes, it is known that any alteration in the availability of SAM may have profound impacts on cellular growth, differentiation, and function [20]. Previous studies as demonstrated in vitro and in vivo have provided evidence that chronic elevation in plasma Hcy associated with vitamins deficiencies have an indirect and negative effect on cellular SAH levels, which should result in feedback inhibition of SAM-dependent methyl-transferase reactions and reduced methylation capacity. This has to lead to the following hypothesis that homocysteine is a metabolic marker for a reduced methylation activity $[9,21,22]$. The proper differentiation potential of Mesenchymal stem cell (MSC) into osteoblasts or adipocytes determines the balance between osteoblast and adipocyte number and activity, which is required to maintain bone and fat homeostasis. Therefore, disturbance of this equilibrium may contribute to the pathology of metabolic diseases such as osteoporosis [23,24]. The mechanisms that determine whether MSC differentiate into osteoblasts or adipocytes are still poorly understood. Recently, Milhem et al. [24] reported that DNA methylation plays an important role in the regulation of hematopoietic stem cell switching, but the link between methylation pathway and MSC differentiation, and whether this effect can be explained by high Hcy remain ambiguous. Therefore, in this study it is hypothesized that the alterations in the commitment and differentiation potential of MSC during osteoporosis may be explained by modification of global methylation pathway. Moreover, to assess the role of demethylation potential in modifying stem cells fate decisions, SAH hydrolase in human MSC lines was inhibited with peroidate oxidized adenosine (Adox) in vitro Figure 1.

\section{Materials and Methods}

\section{Materials}

Culture media (DMEM, aMEM \& hMSCM), fetal bovine serum (FBS), L-glutamine, penicillin /streptomycin, and phosphate buffered saline (PBS) were obtained from (Lonza). Insulin from Novo Nordisk was used, and the remaining tissue culture materials, and periodateoxidized adenosine (adenosine-2, 3-dialdehyde) (Adox) were obtained from Sigma Chemical Company. Netherlands.

\section{Cell culture}

In this study, human Mesenchymal stem cells (hMSCs) derived from human bone marrow, with the potential to differentiate into multiple cell types, for example osteoblasts, adipocytes or chondrocytes dependent on the stimulation agents were used. The cell culture took place in a humidified atmosphere containing $5 \% \mathrm{CO}_{2}$ at $37^{\circ} \mathrm{C}$. After reaching a confluency of $70-80 \%$, all cells were rinsed with $\mathrm{PBS}$ (Lonza), treated with trypsin-EDTA $(0.25 \mathrm{mg} / \mathrm{dl})$ (Cambrex) until cells detached, and subsequently passed at lower density to new flasks in culture medium. Control medium consists of Dulbecco's modified Eagle medium (DMEM) supplemented with 10\% FBS, 2 mM L-glutamine, and $1 \%$ penicillin $(100 \mathrm{U} / \mathrm{ml}) /$ streptomycin $(100 \mu \mathrm{g} / \mathrm{ml})$. The cells were cultured in presence or absence of series concentrations of Adox. Osteoblast differentiation (OB) medium consists of control medium supplemented with $12,5 \mathrm{mM} \beta$-glycerophosphate, $50 \mu \mathrm{g} / \mathrm{ml}$ ascorbic acid and $1 \mathrm{mM}$ dexamethasone. Adipocyte differentiation (AD) medium consists of control medium supplemented with 1 $\mathrm{mM}$ dexamethasone, $5 \mu \mathrm{g} / \mathrm{mL}$ insulin, $111 \mu \mathrm{g} / \mathrm{ml}$ 3-isobutyl-methyl xanthine (IBMX) and $1 \mu \mathrm{M}$ rosiglitazone was added as well. All cell lines in culture medium were seeded in DMEM /10\% FBS at a density of $1.0 \times 10^{4} \mathrm{cells} / \mathrm{cm}^{2}$. Differentiation was initiated two days later (t0) by removing the culture medium and replacing it with either control medium, osteoblast differentiation medium or adipocyte differentiation medium as supplied by the manufacturer. From t0, cells were exposed to series concentrations of Adox.

Alkaline phosphate assay: To measure the progress of osteoblast differentiation, the activity of the protein Alkaline Phosphatase (ALP) assay was measured. Briefly, cells were cultured in 96-wells plates and medium conditions were applied in triplicate. A spectrophotometic analysis of ALP protein activity was performed at various time points. The cells were rinsed with cold PBS, and fixed with $4 \%$ paraformaldehyde for 10 minutes at $4^{\circ} \mathrm{C}$. Subsequently, a dilution of P-nitrophenyl phosphate (pNPP) (Sigma) was used based on the type of cell line. Substrate for ALP was added and the plate was incubated at $37^{\circ} \mathrm{C}$ for 30 minutes. The color reaction, taking place through the conversion of pNPP to p-nitrophenol by ALP, was stopped by addition of $\mathrm{NaOH}$ and the extinction was measured at $405 \mathrm{~nm}$ with a MultiSkan Ascent spectrophotometer (Thermo Labsystems) and version 2.4.2 MultiSkan Ascent software.

Neutral red assay: To correct for cell numbers during the ALP assay, a Neutral Red (NR) assay was carried out. This assay is always performed in combination with ALP assay that so-called ALP/NR assay to correct the cell numbers by dividing the result of the ALP assay by the result of the NR assay. Based on manufacture's protocol, cells were seeded in 96-wells plates and medium conditions were applied in triplicate. Neutral Red (Sigma) $(1 \mathrm{mg} / \mathrm{ml})$ was diluted in PBS, then added to each well of a 96 -wells plate and the plates were incubated at $37^{\circ} \mathrm{C}$ for one hour. Subsequently the cells were rinsed with PBS and the remaining absorbed NR was released by adding an elution solution. Extinction measurements were performed at $550 \mathrm{~nm}$ on a MultiSkan spectrophotometer.

Alkaline phosphatase/Oil-red-O staining: To visualize the amount of osteoblasts and adipocytes, alkaline phosphatase/Oil-red-O staining (ALP/ORO) was used. Briefly, Oil-red-O staining was performed on distinct time points. The cells were fixed for 30-60 seconds with citratebuffered acetone (kit from Sigma-Aldrich) and then washed twice with MilliQ water (MQ). ALP staining was performed by adding a dye mixture of diazonium salt solution and Naphtol AS-MX phosphate alkaline solution (Sigma-Aldrich). The cells were incubated in the dark for 30 minutes to 3 hours at $37^{\circ} \mathrm{C}$ until the blue staining was visible. After which they were rinsed with MQ water. For subsequent lipid droplets staining, an Oil-Red-O (Sigma) working solution was added and the plate was incubated for 15 minutes at $37^{\circ} \mathrm{C}$. The cells were rinsed once again with $\mathrm{MQ}$ and photographs were taken under the microscope (Olympus CKX41) using an Olympus C-5050 Zoom camera and Olympus DP-soft version 3.2 software.

Calcium release assay (quantification of calcification): This assay is performed to measure the concentration of calcium which is formed during the later stage of osteoblast differentiation as an indicator for the level of calcification or mineralization. Cells were cultured in 24-wells plates and medium conditions were applied. Calcium deposition was measured with a calcium release assay when the whole time-series was isolated. The cells were incubated with HCL $(0.5 \mathrm{M})$ and shaken overnight. A color reaction was performed by adding $25 \mu \mathrm{l}$ of the collected and centrifuged HCL samples to a mix of o-cresolphthalein complexone, 8-quinolinol and 2- amino-2-methyl-1, 3-propanediol (Sigma) in cuvettes. A blank $(25 \mu \mathrm{l} \mathrm{HCL})$ and a standard series of 
calcium solution, were included. Extinction was determined at $575 \mathrm{~nm}$ on a UV mini 1240 spectrophotometer (Shimadzu).

Triglyceride assay (quantification of lipid): A triglyceride assay was performed to measure the degree of adipocyte differentiation and lipid accumulation. Since, adipocytes are a highly specialized cell type included in the store and accumulate lipid as triglyceride in response to adipogenic factors such as insulin, dexamethasone, rosiglitazone and IBMX. Briefly, cells were seeded in 24-wells plates and medium conditions were applied in duplicate followed by rinsing with PBS, then incubated with a Tris- $\mathrm{HCl}$ EDTA solution at -20 until all samples were collected.

Plates were brought to room temperature to determine triglyceride content according to the protocol. A standard series was included by adding triglyceride standard (kit from iNstrucchemie, ref \# 10720P) to wells without cells. $40 \mu \mathrm{l}$ Tertiary butanol and $10 \mu \mathrm{l}$ methanol were added to the wells to extract the triglycerides from the cells, and shaking for 10 minutes. A color reaction was performed by adding the reagent triglycerides Liquicolour Mono (kit from iNstruchemie, ref \#10720P). After shaking for 20 minutes, samples were pipetted into a 96-wells plate and extinction was measured at $492 \mathrm{~nm}$ with a MultiSkan spectrophotometer.

\section{Statistical Analysis}

Data are expressed as the mean \pm standard deviation. One wayANOVA (SPSS version 20.0) and LSD post hoc test were used to determine the mean differences between groups. Values are considered significantly different at the level of $<0.05$.

\section{Results}

\section{Osteoblast differentiation}

Continuous treatment the confluent cells with differentiation inducers resulted in terminal oseoblast differentiation. Adox was used as a potent methylation inhibitor because it has been shown to inhibit methylation pathway in previous studies [13,21].

\section{ALP staining}

Cells were stained with alkaline-dye to visualize the amount of differentiated osteoblasts from MSCs. Cells were cultured in control medium without any supplements and $\mathrm{OB}$ medium supplemented with (ascorbic acid, beta-glycerophosphae, vitamin D and dexamethasone) in the presence of series concentrations of Adox $(5,25$ and $50 \mu \mathrm{M})$ and then stained for ALP-positive cells using ALP staining on day 7 , the resulting pictures are presented in Figure 2.

The blue staining indicates osteoblast differentiation. The pictures show that in $\mathrm{OB}$ medium large numbers of the human stem cells were differentiated into osteoblasts that express high amount of ALP compared with those in control medium due to the potent effect of osteogenic supplements that were used for osteoblast differentiation. A marked decrease in the number of ALP-positive cells treated with series concentrations of Adox compared with the number of positive cells in untreated osteogenic medium (OB) can be observed. This result indicates that osteogenesis is decreased due to the inhibition of methylation pathway in hMSCs.

\section{Alkaline phosphatase activity}

To quantify the result of osteoblast differentiation in cells, ALP assay was done. Cells were treated with different concentrations of Adox $(5,25$ and $50 \mu \mathrm{M})$. The influence of Adox on the synthesis of ALP by osteoblasts was determined by measuring ALP activity, corrected for cell number as determined by neutral red adsorption. ALP activity was measured on day 6, day 10 and day 14 respectively, and the resulting graph is shown in Figure 3. As observed by spectrophotometric measurement, ALP activity is strongly induced in osteogenic medium compared with control medium. A marked decrease in the ALP activity compared with the ALP activity in osteogenic medium can be observed in the cells that have been treated with series concentrations of Adox.

\section{Calcification}

To investigate the effect of inhibition of the methylation pathway by Adox on matrix calcification or mineralization, a calcium release assay was performed in cells. Cells were grown in control and osteogenic medium for 20 days and the calcium release assay was measured on t11, t17, and t20. From the results shown in Figure 4, it can be stated clearly that osteogenic agents have stimulatory effect on calcium release in osteogenic medium compared with control medium.

While, Adox in treated cultures has strong an inhibitory effect on calcium release at every time points compared with osteogenic medium. A dramatic repression is achieved on exposure to $50 \mu \mathrm{M}$ of Adox, which disables the cells to calcify at all.

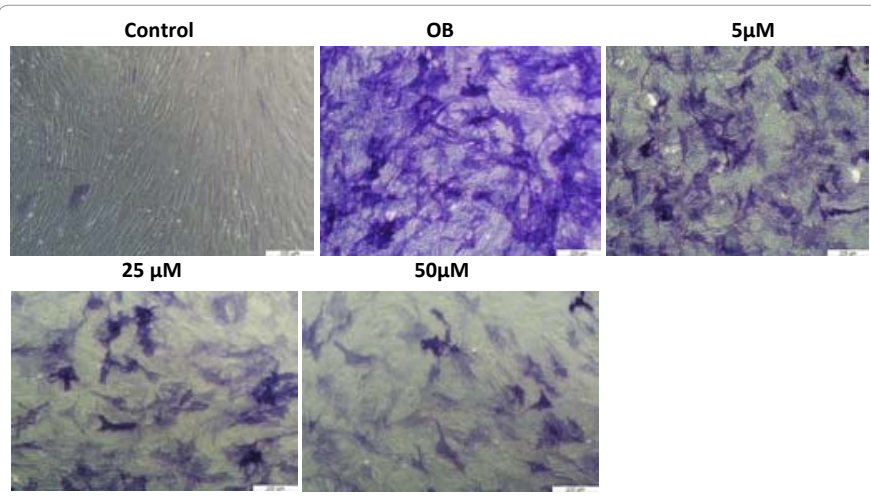

Figure 2: Photographs of hMSC cells treated with Adox on day 7. All cells were stained with alkaline-dye, according to the protocol described in material and methods. All cells in the control were only exposed to control medium without any osteogenic supplements, the other cells were cultured in OB medium containing different concentrations of Adox. 10,000x magnification (scale bar is $200 \mu \mathrm{m})$. OB, osteogenic medium.

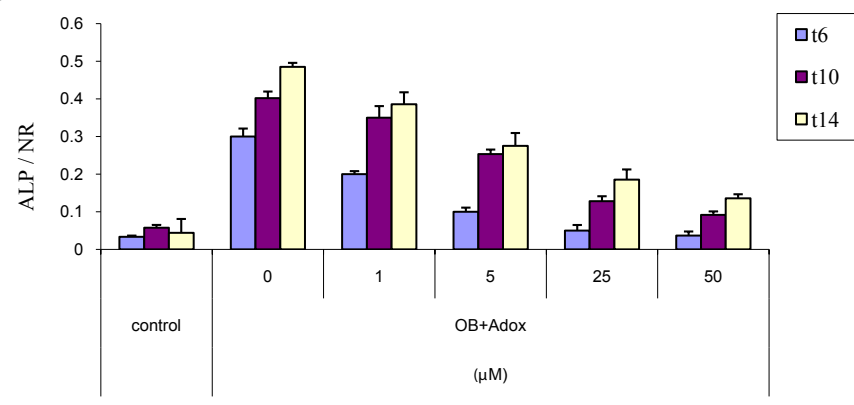

Figure 3: ALP/NR assays of hMSCs treated with Adox. The assays were performed on $\mathrm{t} 6$ and $\mathrm{t} 10$ and $\mathrm{t} 14$ respectively, according to the protocol in materials and methods. Cells in the control condition were only exposed to control medium without any osteogenic supplements, the other cells were cultured in OB medium. Results are expressed as means $\pm S D(n=3)$. Differences between means were significantly different $p<0.01$. Error bars indicate standard deviations. 


\section{Adipocyte differentiation}

The confluent cells were cultured in control medium lacking adipogenic agents and in adipogenic medium containing differentiation inducers dexamethasone, insulin, rosiglitazone and isobutylmethylxanthine. Cells treated with different concentrations of Adox $(5,25$ and $50 \mu \mathrm{M})$ and then stained for lipid droplets using an ORO staining on day 9, the resulting pictures are presented in Figure 5.

\section{ORO staining}

The red spots indicate accumulation of lipid droplets, serving as an indication of adipocyte differentiation. The pictures clearly show that in adipogenic medium, hMSC were differentiated into adipocytes that produce lipid droplets compared with those in control medium due to the potent effect of adipogenic supplements that were used for adipogenic differentiation. However, higher numbers of human stem cells were differentiated into adipocytes in the cells that have been treated with series concentration of Adox compared with Ad medium lacking Adox treatment. Interestingly, the treated cells with a higher concentration of Adox have been filled with an extensive accumulation of lipid droplets compared with adipocytes in Ad medium.

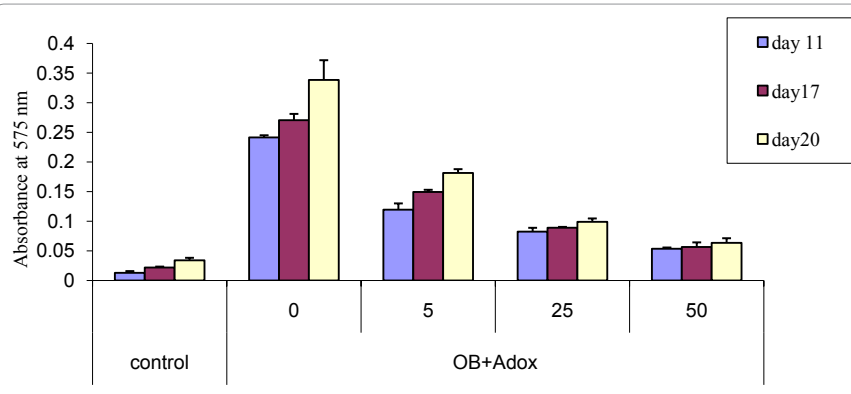

Figure 4: Calcium release assay of hMSCs treated with Adox. The assays were performed on day 11 , day 17 and day 20 respectively, according to the protocol in materials and methods. Cells in the control condition were only exposed to control medium without any osteogenic supplements, the other cells were cultured in OB medium. Results are expressed as means $\pm S D(n=3)$. Differences between means were significantly different $p<0.01$. Error bars indicate standard deviations.

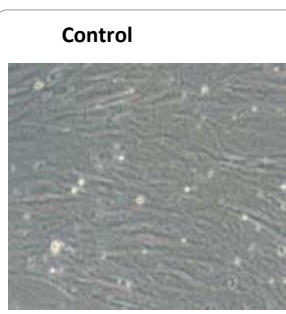

$25 \mu \mathrm{M}$

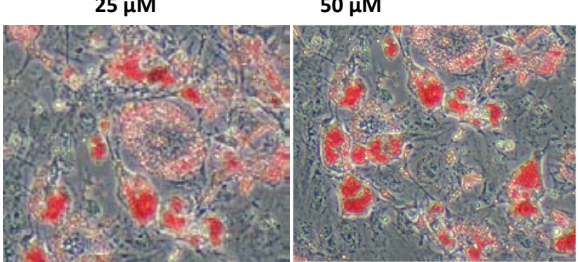

Figure 5: Photographs of hMSC cells treated with Adox on day 9. All cells were stained with ORO, according to the protocol described in Material and Methods. All cells in the control were only exposed to control medium without any adipognic supplements, the other cells were cultured in Ad medium containing different concentrations of Adox. 10,000x magnification (scale bar is $200 \mu \mathrm{m}$ ). Ad, adipogenic medium without Adox treatment.

\section{Level of triglyceride}

To quantify the result of adipocyte differentiation of hMSC, a triglyceride assay was carried out. The result is shown in Figure 6, and a great similarity with ORO staining can be observed especially at ( $\mathrm{t} 7$ and t9). At every time point a positive effect of adipogenic agents on adipocyte differentiation was seen compared with control medium. Whereas, Adox treatment clearly results in induction of the amount of triglycerides in a dose dependent way in treated cells compared with those in adipogenic medium.

\section{Osteoblast and adipocyte differentiation}

We next studied the effect of Adox on osteogenesis and adipogenesis simultaneously to assess the role of methylation pathway on the osteoblast and adipocyte differentiation together. The hMSCs were used and stained with alkaline-dye mixture and ORO staining. The result is shown in Figures 7 respectively. The blue staining indicates osteoblast differentiation. While, the red spots indicate accumulation of lipid droplets inside adipocyte differentiation and the medium was meant to trigger adipogenesis. Although, the cells were grown in adipogenic medium osteoblasts formation were present.

\section{Discussion}

In the present study we have investigated the role of demethylation in commitment and cellular differentiation of multipotent stem cells as characterized by ALP/NR, calcium release, triglyceride assays and ALP/ORO staining. It is clearly shown that demethylation reduces stem cells differentiation into mature osteoblasts and therefore, inhibits osteogenesis in human stem cells; this indicates that demethylation act as a negative regulator of osteoblast differentiation. Moreover, this study demonstrated that the inhibitory effect of demethylation is not restricted on the early stage of stem cells but also this effect persists throughout the later stage of osteoblast differentiation as has been shown in the level of calcification that already committed to osteoblast lineage Figure 4. The results obtained from calcium release in hMSCs were very interesting and showed that demethylation also inhibits mineralization of matrix proteins that is the major function of osteoblast. On the other hand, demethylation obviously enhances stem cells differentiation into mature adipocytes and therefore, induces adipogenesis in hMSC. This result is in line with other study performed in embryonic stem cells reported that DNA hypomethylation associated with lipid deposition in the proximal portion of the aorta of mice in vivo [25]. But, C3H10T1/2 cells don not show any stimulatory or inhibitory effect under the influence of demethylation in the presence of rosiglitazone. However, another study showed that blocking DNA methylation by using another methylation inhibitor called Aza in C3H10T1/2 stem cells without roziglitazone, resulted in stimulation of adipogenesis after culturing for 28 days [26].

One of the explanations is that adipocte differentiation was very strong due to the strong effect of rosiglitazone a potent inducer of adipocyte differentiation. That means if Adox would stimulate adipocyte formation it cannot be detected. Furthermore, the influence of demethylation on osteogenesis and adipogenesis were studied simultaneously Figure 7. It was observed that demethylation is associated with a reciprocal decrease of osteogenesis and an increase of adipogenesis. These results clearly show the role of methylation pathway as a key regulator of stem cell differentiation switch into less osteogenic and more adipogenic cells. Since, demethylated stem cells failed to differentiate into osteoblasts but differentiated into adipocytes in OB medium of stem cells, which has been used to trigger osteogenesis, 


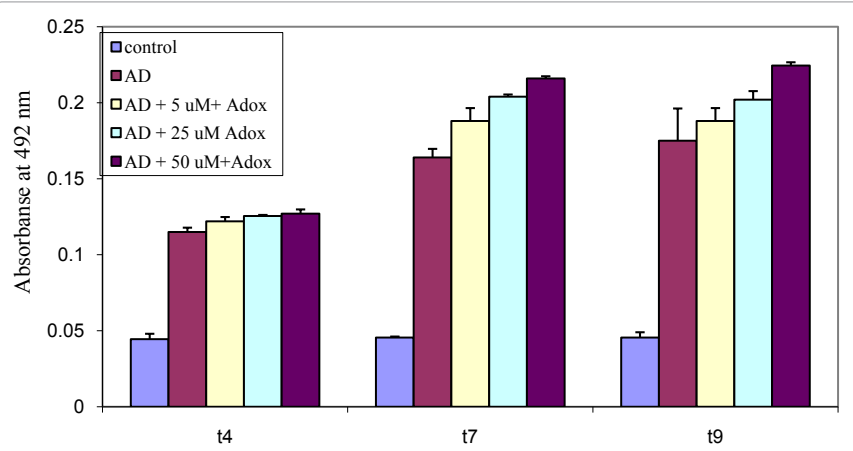

Figure 6: Triglyceride of hMSC cells treated with Adox on day 4, day7 and day9 respectively. All measurements were done as duplicate and averaged, according to the protocol described in Material and methods. All cells in the control were only exposed to control medium, the other cells were cultured in Ad medium containing different concentrations of Adox. Results are expressed as means $\pm S D(n=3)$. Differences between means were significantly different $p<0.01$ Error bars indicate standard deviations.

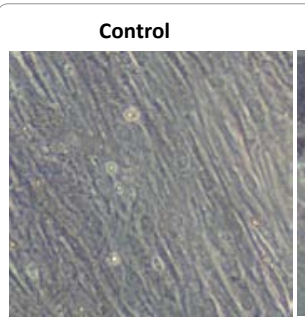

$5 \mu \mathrm{M}$

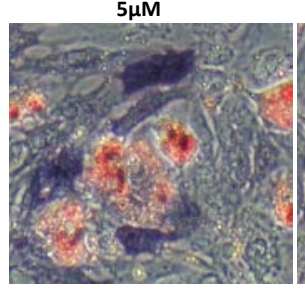

Figure 7: Photographs of hMSC cells treated with Adox on day 11. All cells were stained with alkaline-dye mixture and Oil-red-O, according the protocol described in material and methods. All cells in the control were only exposed to control medium without any adipogenic medium, the other cells were cultured in Ad medium containing different concentrations of Adox.10,000x magnification (scale bar is $200 \mu \mathrm{m}$ ).

the high degree of adipogenesis was unexpected. The inverse relation between the number of osteoblasts and the number of adipocytes under the effect of demethylation reveals the importance of the methylation pathway in MSC for proper commitment and differentiation. In addition, the role of methylation pathway as a key regulator for stem cell differentiation is also observed in hematopoietic stem cell (HSC) [27] after being treated with Aza a potent DNA methylation inhibitor and TSA as histone deacetylation inhibitor resulting in a significant alteration in the fate of HSC [28].

These findings support the concepts that methylation modification may play an important role in the differentiation switching of stem cells. In this study it must be mentioned that the present results of osteoblast differentiation by our model systems are not in agreement with previous studies that showed that DNA demethylation by Aza agent in hMSC and MG63 cells is associated with elevation of ALP expression and mineralization and therefore, osteoblast differentiation [28,29]. A possible explanation for the lowered osteoblast differentiation in our study after treated the cells with Adox could be resulted from demethylation of protein, lipid or RNA but DNA methylation might not be involved in the negative effect of demethylation. Since, that DNA methylation is not influenced by short-term alterations in methylation potential as has been observed previously by [30], who reported that a reduced methylation potential in HepG2 cells by Adox does not change the global DNA methylation while, mRNA methylation is reduced within $24 \mathrm{~h}$. Moreover, others showed a clear reduction in protein methylation after Adox treatment [31,32]. However, in our study we do not examine global DNA methylation but based on the literatures we propose that DNA methylation could be not changed via inhibition of methyl acceptors that are involved in the process of cellular differentiation of MSC. Since, Adox was used to block methylation of DNA, RNA, lipid and protein indirectly by inhibition of SAH hydrolase enzyme in methylation cycle, therefore, it is not known exactly which methyl acceptor is inhibited and caused reduction for osteoblast differentiation. But, Aza in the previous studies has been directly incorporated into DNA molecule and thereby prevent methylation of DNA in the replicated stem cells.

Therefore, further studies are needed to clarify the influence of an altered of methylation pathway on DNA, mRNA or protein methylation separately to elucidate whether the effect is due to a changed methylation of DNA, mRNA or due to the modification of protein methylation. Furthermore, determination of SAM, SAH and methylation levels are recommended as parameters for cellular methylation status.

\section{Conclusion}

In summary, the results of this study suggest that demethylation changes the differentiation potential of MSC for more adipogenic and less osteogenic. Since inhibition of methylation pathway in hMSC by Adox induced adipocyte differentiation and reduced osteoblast differentiation. Moreover, we can conclude that methylation pathway may play a pivotal role during stem cells commitment and differentiation and able to determine whether stem cells differentiate into osteoblasts or adipocytes. Thereby, our results support the hypothesis that alteration in the balance between osteoblast and adipocyte differentiation during osteoporosis and aging might be mediated by demethylation. Finally, it may be possible to regulate osteoblats and adipocyte differentiation in humans pharmacologically by using a specific modulator of methylation pathway which could open new strategy for therapeutic intervention of osteopenic disorders such as osteoporosis.

\section{Acknowledgment}

The authors are very grateful to the department of Nutrition and Dietetics, Faculty of Medicine and Health Sciences and RMC at UPM for their assistance in English editing and for allowing us to publish this manuscript. This study was supported by (Netherlands Fellowship Programme, Nuffic).

\section{References}

1. Afman LA, Blom HJ, Drittij MJ, Brouns MR, van Straaten HW (2005) Inhibition of transmethylation disturbs neurulation in chick embryos. Brain Res De 158 : 59-65.

2. Afman LA, Blom HJ, van der Put NM, van Straaten HW (2003) Homocysteine interference in neurulation : a chick embryo model. Birth Defects Res A Clin Mol Teratol 67: 421-428.

3. Bainbridge KE, Sowers M, Lin X, Harlow SD (2004) Risk factors for low bone mineral density and the 6-year rate of bone loss among premenopausal and perimenopausal women. Osteoporos Int 15: 439-446.

4. Boer JD, Licht R, Bongers M, van der Klundert T, Arends R, et al. (2006) Inhibition of histone acetylation as a tool in bone tissue engineerng. Tissue Eng 12: 2927-2937. 
Citation: Ali F, Ranneh Y, Ismail A, Vaes B (2013) Influence of Demethylation Pathway on the Differentiation Potential of Human Mesenchymal Stem Cells. J Stem Cell Res Ther 3: 153. doi:10.4172/2157-7633.1000153

5. Chiang PK, Gordon RK, Tal J, Zeng GC, Doctor BP, et al. (1996) S-adenosylmethionine and methylation. FASEB J 10: 471-480.

6. Chen Z, Karaplis AC, Ackerman SL, Pogribny IP, Melnyk S, et al. (2001) Mice deficient in methylenetetrahydrofolate reductase exhibit hyperhomocysteinia and decreased methylation capacity, with neuropathology and aortic lipid deposition. Hum Mol Genet 10: 433-443.

7. Carmel R, Lau KH, Baylink DJ, Saxena S, Singer FR (1988) Cobalamin and osteoblast -specific proteins. N Engl J Med 319: 70-75.

8. Caudill MA, Wang JC, Melnyk S, Pogribny IP, Jemigan S, et al. (2001) Intracellular S-adenosylhomocysteine concentrations predict global DNA hypomethylation in tissues of methyl-deficient cysta-thionine (beta)-synthase heterozygous mice. J Nutr 131: 2811-2818.

9. Duk JM, Jung MK, Oksun L, Na Jung K, Young SL, et al. (2006) Homocysteine enhances apoptosis in human bone marrow stromal cells. Bone 39: 582-590.

10. Dhnukshe-Rutten RA, Pluijm SM, de Groot LC, Lips p, Smit JH, et al. (2005) Homocysteine and vitamin B12 status relate to bone turnover markers broadband ultrasound attenuation, and fractures in healthy elderly people. $J$ Bone Miner Res 20: 921-929.

11. Dhanukshe-Rutten RA, Lips M, de Jong N, Chin A Paw MJ, Hiddink GJ, et al (2003) Vitamin B12 status is associated with bone mineral content and bone mineral density in frail elderly women but not in man. J Nutr 133: 801-807.

12. Delong CJ, Hicks AM, Cui Z (2002) Disruption of choline methyl group donation for phosphatidylethanolamine methylaton in hepatocarcinoma cells. J Biol Chem 277: 17217-17225

13. Moerman EJ, Teng K, Lipschitz DA, Lecka-Czernik B (2004) Aging activates adipogenic and suppresses osteogenic programs in mesenchymal marrow stoma/stem cells: the role of PPAR-gamma2 transcription facto and TGF- $\beta$ BMP signaling pathways. Aging cell 3: 379-389.

14. Gjesdal CG, vollset SE, Veland PM, Refsum H, Meyer HE, et al. (2007) Plasma homocysteine, folate, vitamin B12 and the risk of hip fracture. J Bone Miner Res 22: 747-756.

15. Hoffman DR, Marion DW, Cornatzer WE, Duerre JA (1980) S-adenosylmethionine metabolism and S-adenosylhomocysteine in isolated rat liver. Effects of L-methionine, L- homocysteine and adenosine. J Biol Chem 255: 10822-10827.

16. Jeremy B, John T, Lubert S (2003) Biosynthesis of amino acids. Biochemistry Chapter 24: 675-679.

17. Koh JM, Lee YS, Kim YS, Kim DJ, Kim HH, et al. (2006) Homocysteine enhances bone resorption by stimulation of osteoclst formation and activity through increased intracellular ROS generation. J Bone Miner Res 21: 10031011.

18. Li C, Ai LS, Lin CH, Hsiek M, Li YC, et al. (1998) Protein N-arginine methylation in adenosine dialdehyde-treated lymphoblastic cells. Arch Biochem. Biophys 351: 53-59.

19. Markus H, Thomas W, Graziana C, Silvia C, Alberta Z, et al. (2005) Increased osteoclast activity in the presence of increased homocysteine concentrations. Clin Chem 51: 2348-2353.

20. Markus H, Peter Schmidt J, Umanskaya N, Wagner A, Taban-Shomal O, et al. (2007) The role of hyperhomocysteinemia as well as folate, vitamin B6 and B12 deficiencies in osteoporosis. Clin Chem Lab Med 45: 1621-1632.

21. Markus H, Umanskaya N, Wildemann B, Colaianni G, Schmidt J, et al. (2007) Accumulation of homocysteine by decreasing concentrations of folate, vitamin B12 and B6 does not influence the activity of human osteoblasts in vitro. Clin Chim Acta 384: 1229-1234

22. Marina H, Hartmut O, Julia M, Doris K (2004) Influence of an altered methylation potential on mRNA methylation and gene expression in HepG2 cells. Exp Cell Res 29: 325-334.

23. Marina H, Hippel von S, Osswald H, Kloor D (2005) S-adenosylhomocysteine metabolism in different cell lines: effect of hypoxia and cell density. Cell Physio Biochem 15: 233-244.
24. Milhem M, Mohmud N, Lavelle D, Araki H, Desimone J, et al. (2004) Modification of hematopoietic stem cell fate by 5aza 2' deoxycytidine and trichostatin A Blood 11: 4102-4109.

25. Ping YI, Melnyk S, Pogribna M, Pogribny IP, Hiner RJ, et al. (2000) Increase in plasma homocysteine associated with parallel increases in plasma S-adenosylhomocysteine and lymphocyte DNA hypomethylation. J Bio Chem 275: 29318-29323.

26. Castro R, Rivera I, Struys EA, Jansen EE, Ravasco P, et al. (2003) Increased homocysteine and S-adenosylhomocysteine concentrations and DNA hypomethylation in vascular disease. Clin Chem 49: 1292-1296.

27. Ralph Carmel, Jacobsen DW (2001) Homocysteine in health and disease. Chapter 4: Homocysteine in health and disease 52-110.

28. Locklin RM, Oreffo RO, Triffitt JT (1998) Modulation of osteogenic differentiation in human skeletal cells in vitro by 5-Azacytidine. Cell Bio Inter 3: 207-215.

29. Seema B, Parakh R, Srivastava LM (2004) Studies on homocysteine demonstrating its significance a possible tool for differential diagnosis in occlusive vascular disease. Indian J Clin Biochem 19: 76-78.

30. Taylor SM, Jones PA (1979) Multiple new phenotype induced in T101/2 and MC3T3 cells treated with 5-azacytidine. Cell 17: 771-779.

31. Akune T, Ohba S, Kamekura S, Yamaguchi M, Chung UI, et al. (2004) PPAR gamma insufficiency enhances osteogenesis through osteoblast formation from bone marrow progenitors. J Clin Invest 113: 846-855.

32. Van Meurs JB, Dhnukshe-Rutten RA, Pluijm SM, van der Klift M, de Jonge R, et al. (2004) Homocysteine levels and the risk of osteoporotic fracture. N Eng J Med 350: 2033-2040. 